\title{
The synthesis of 4-aryl-1H-pyrazolo[3,4-b]quinolines by cyclization of 4-arylidenepyrazolin-5-ones with anilines
}

\author{
Karen Chaczatrian, Gohar Chaczatrian, Andrzej Danel, and Piotr Tomasik* \\ Department of Chemistry, University of Agriculture Mickiewicz Ave. 24/28, 30-059 \\ Cracow, Poland \\ E-mail: rrtomasi@cyf-kr.edu.pl
}

(received 28 Jun 01; accepted 24 Sep 01; published on the web 02 Oct 01)

\begin{abstract}
In the reaction of anilines with 4-(benzylidene)-1,3-disubstituted pyrazol-5-ones, $1 H$ pyrazolo[3,4-b]quinolines were formed instead of anticipated 5-phenylimino derivatives. The same compounds were also available from the reaction of pyrazolin-5-ones with Schiff bases. In spite of moderate yields both methods present a facile route to 4-aryl-1H-pyrazolo[3,4b]quinolines. These methods have advantages over the Friedländer cyclization commonly utilized for preparation of 4-aryl-1H-pyrazolo[3,4-b]quinolines. Thus, preparation of 2aminobenzophenones, reagents indispensable in Friedlaender synthesis, can be avoided.
\end{abstract}

Keywords: $1 H$-Pyrazolo[3,4-b]quinolines, luminophores, pyrazolin-5-ones

\section{Introduction}

In 1928 Michaelis $^{1}$ reported that heating of 5-phenyliminopyrazoles $\mathbf{1}$ with aromatic aldehydes 2 resulted in the formation of 4-benzylidene-5-phenyliminopyrazoles 3 (Scheme 1).

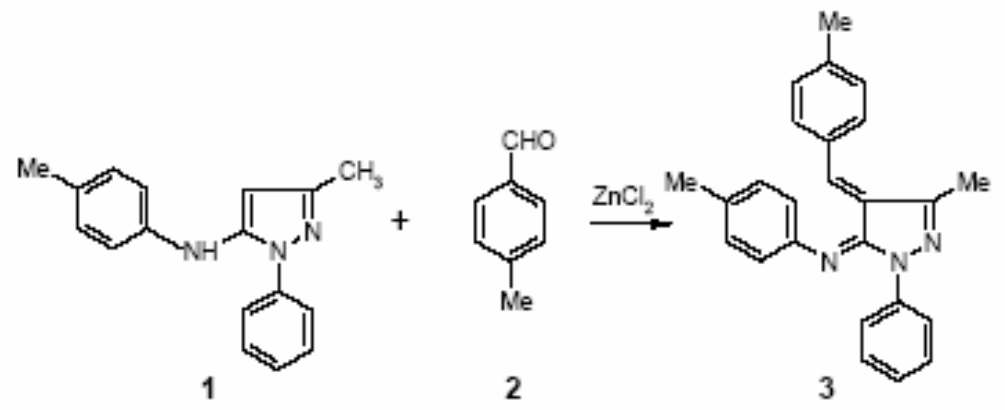

\section{Scheme 1}

Products of this reaction exhibited a strong blue fluorescence in solution. We considered this 
method as an appropriate, facile route providing a series of novel fluorophores for electroluminescent devices. A careful examination of the products of this reaction by ${ }^{1} \mathrm{H}$ NMR indicated that Michaelis synthesized $1 H$-pyrazolo[3,4-b]quinolines (4). Thus, this synthesis appears to be very convenient for preparation of 4-arylpyrazolo[3,4-b]quinolines. ${ }^{2}$. Friedländer condensation as an alternative route to such compounds involves 2-aminobenzophenones and pyrazolin-5-ones, ${ }^{3,4}$ however availability of 2 -aminobenzophenones limits the range of applicability of this reaction.

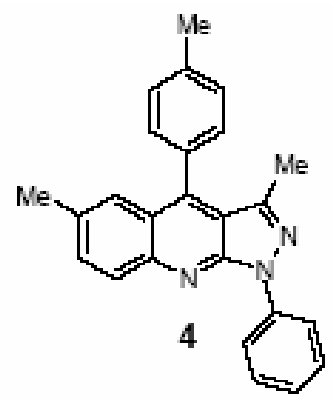

These results prompted us to look at the synthesis of 4-arylidene-5-aryliminopyrazoles 7 as promising intermediates for preparation of corresponding 4-aryl-1H-pyrazolo[3,4-b]quinolines (8). Thus, synthesis of the benzylidene compounds 7 was attempted by a heating an equimolar mixture of 4-(arylidene)-pyrazolin-5-ones (5) and aromatic amine 6 (Scheme 2).

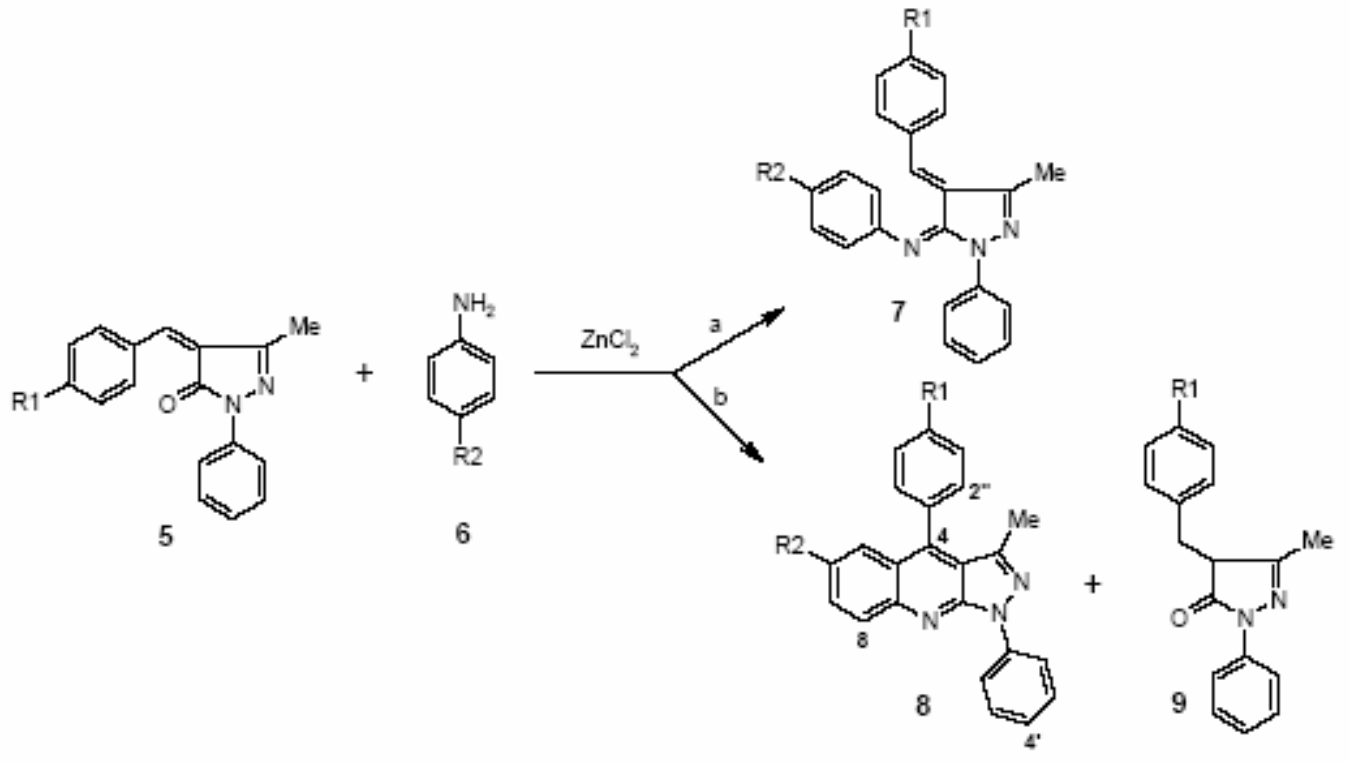

Scheme 2. $\mathrm{R}^{1}=\mathrm{OMe} ; \mathrm{R}^{2}=\mathrm{OMe}(\mathbf{8 a}), \mathrm{H}(\mathbf{8 b}), \mathrm{Me}(\mathbf{8 c})$, tert-Bu $(\mathbf{8 d}), \mathrm{F}(\mathbf{8 e}), \mathrm{Cl}(\mathbf{8 f}), \mathrm{Br}(\mathbf{8 g}) ; \mathrm{R}^{1}$ $=\mathrm{Me} ; \mathrm{R}^{2}=\mathrm{H}(\mathbf{8 h}), \mathrm{Me}(\mathbf{8 i}), \mathrm{Et}(\mathbf{8 j}), n-\mathrm{Bu}(\mathbf{8 k}), \mathrm{F}(\mathbf{8 i}), \mathrm{Cl}(\mathbf{8 m}), \mathrm{Br}(\mathbf{8 n}), \mathrm{OMe}(\mathbf{8 o}), \mathrm{OPh}(\mathbf{8 p})$.

Results of this synthesis are described in this paper. When the first attempt to prepare 4arylidene-5-phenyliminopyrazole failed we tried another way by condensing 4-arylidenepyrazol5-one with anilines in the presence of anhydrous zinc chloride. But again in spite of our efforts the only resulted product was pyrazolo[3,4-b]quinolines and reduced benzylidene system. The 
same product was obtained when the reaction was done without $\mathrm{ZnCl}_{2}$. The yields of pyrazolo[3,4-b]quinolines never exceed $31-32 \%$.

\section{Results and Discussion}

As in the Michaelis approach $^{1}$ the attempted preparation of 5-phenylimino-4benzylidenepyrazolin-5-ones failed. Regardless of the nature of substituents R1 and R2 on substrates, and application of various condensation agents $\left(\mathrm{ZnCl}_{2}\right.$ or phosphorus pentoxide in xylene) 4-aryl-1H-pyrazolo[3,4-b]quinolines 8 were formed. They were accompanied by 4methylarylpyrazolin-5-one 9 as the product of reduction of corresponding benzylidenepyrazolin5-ones. These products were isolated in the ratio 1:1 that provided evidence that the corresponding benzylidene compounds are one of the partners of condensation with anilines acted as oxidants for 4-benzylidene-5-aryliminopyrazolines 7. This reaction limits the overall yield of $\mathbf{8}$. The structures of both series were confirmed by results of NMR measurements and elemental analysis. The 4-(4'-methoxyphenyl)-pyrazolo[3,4-b]quinolines were also prepared ${ }^{5}$ by reaction of substituted anilines and 4-(4'-anisoyl-5-chloro-3-methyl-1-phenylpyrazole. The NMR spectra for products of both reactions were identical. Results of electroluminescent studies of 4(4'-methoxyphenyl) derivatives were published recently. ${ }^{5}$ Such data for $4-\left(4^{\prime}\right.$-methylphenyl) series are collected in Table 1.

Table 1. Luminescence of 4-(4'-methylphenyl) derivatives of $1 H$-pyrazolo[3,4-b]quinolines

\begin{tabular}{ccccc}
\hline Compound & Cyclohexane & Dichloromethane & Ethanol & Acetonitrile \\
\hline $\mathbf{8 h}$ & 437 & 463 & 458 & 467.5 \\
$\mathbf{8 i}$ & 438.5 & 455.5 & 452 & 458.5 \\
$\mathbf{8 j}$ & 437 & 449 & 449 & 453 \\
$\mathbf{8 k}$ & 437 & 448 & 449 & 452 \\
$\mathbf{8}$ & 444.5 & 468.5 & 466.5 & 479 \\
$\mathbf{8 m}$ & 446 & 471 & 470 & 478.5 \\
$\mathbf{8 n}$ & 447 & 468.5 & 471 & 482 \\
$\mathbf{8 0}$ & 448.5 & 458 & 456.5 & 458.5 \\
$\mathbf{8 p}$ & 446.5 & 460.5 & 455 & 465.5 \\
\hline
\end{tabular}

Sensitivity of the pyrazoloquinoline system to the substituent effect from the 6-position expressed in terms of a shift of the longest-wavelength spectral band is low. The largest shift of the emission band in respect to 6-unsubstituted compound reached $11.5 \mathrm{~nm}$. The methoxy group was the most spectrally active. Such moderate band shifts are common for the spectra of compounds with extended chromophore systems, for instance, compounds with three fused aromatic rings such as anthracenes, phenanthrenes, carbazoles. ${ }^{7}$ Simultaneously, a shift of maximum of luminescence up to $35 \mathrm{~nm}$ could be afforded with involvement of the solvent effect. 
A considerably strong solvatochromic effect noted for the parent pyrazoloquinoline increased in a consequence of substitution with halogen substituents $(\mathrm{F}, \mathrm{Cl}, \mathrm{Br})$ but it was significantly reduced by alkyl and alkoxy (phenoxy) substituents. Order of solvents arranged according to increasing bathochromic shift of the luminescence band although slightly varying with substituents clearly showed that nonpolar solvent - solute interactions in solution had a priority.

\section{Experimental Section}

4-(Arylidene)-3-methyl-1-phenyl-pyrazolin-5-ones were prepared according to literature procedures. ${ }^{6}$

Synthesis of 4-(4'-methoxyphenyl)-3-methyl-6-substituted-1-phenyl-1H-pyrazolo[3,4-b]quinolines. General procedure

Equimolar amount of 4-(4'-methoxyphenyl)-3-methyl-1-phenyl-pyrazolin-5-one and substituted aniline $(0.01 \mathrm{~mole})$ were heated for $210 \mathrm{~min}$ in ethylene glycol $(10 \mathrm{~mL})$ at $180{ }^{\circ} \mathrm{C}$. After cooling the solution was digested with methanol $(20 \mathrm{~mL})$ and refrigerated. Insoluble yellow residue was filtered off and methanolic filtrate was added to $10 \% \mathrm{NaOH}(100 \mathrm{~mL})$. The precipitate was dried and subjected to column chromatography (aluminium oxide 90, Merck, chloroform as eluent). After chromatography solution was evaporated and the residue was crystallized from toluene to give 6-methoxy-3-methyl-4-(4'-methoxyphenyl)-1-phenylpyrazolo[3,4-b]quinoline as yellow needless $(1.1 \mathrm{~g}, 31.3 \%)$. The $\mathrm{NaOH}$ solution was extracted 2 times with toluene $(25 \mathrm{~mL})$. The $\mathrm{NaOH}$ solution was boiled with charcoal, filtered and acidified with $10 \%$ hydrochloric acid. The precipitate was filtered, dried and crystallized from ethanol/water yielded 4-(4'-methoxybenzal)3-methyl-1-phenylpyrazolin-5-one as colorless needless (0.75 g, $30.2 \%)$. Toluene solution contained unreacted 4-methoxyaniline.

Pyrazolo[3,4-b]quinolines from the reaction of 4- substituted anilines with 4-(4'methoxyphenyl)-pyrazolin-5-one

6-Methoxy-4-(4'-methoxyphenyl)-3-methyl-1-phenyl-1H-pyrazolo[3,4-b]quinoline (8a). Yellow crystals; $31.3 \%$; m.p. $174-5{ }^{\circ} \mathrm{C}$ (Lit. $\left.{ }^{5} 174.5{ }^{\circ} \mathrm{C}\right) .{ }^{1} \mathrm{H}$ NMR $\left(300 \mathrm{MHz}, \mathrm{CDCl}_{3}\right): \delta 8.50(2 \mathrm{H}$, d, $\left.J=7.7 \mathrm{~Hz}, 2^{\prime}-\mathrm{H}, 6^{\prime}-\mathrm{H}\right), 8.05(1 \mathrm{H}, \mathrm{d}, J=9.34 \mathrm{~Hz}, 8-\mathrm{H}), 7.54$ (2H, t, $J=7.8 \mathrm{~Hz}, 3$ '-H, 5'-H), 7.25 (1H, t, $J=7.4 \mathrm{~Hz}, 4$ '-H), 7.10 (2H, d, $J=8.8 \mathrm{~Hz}$, Harom), 6.93 (1H, d, J=3.02 Hz, 5-H), $3.93(3 \mathrm{H}, \mathrm{s}, \mathrm{OMe}), 3.70$ (3H, s, OMe), 2.15 (3H, s, 3-Me).

4-(4'-Methoxyphenyl)-3-methyl-1-phenyl-1H-pyrazolo[3,4-b]quinoline $(8 \mathrm{~b})$. Yellow needles, 30\%, m.p. $205-7{ }^{\circ} \mathrm{C}$ (Lit. $\left.{ }^{5} 205-7{ }^{\circ} \mathrm{C}\right) .{ }^{1} \mathrm{H}$ NMR (300 MHz, $\left.\mathrm{CDCl}_{3}\right): \delta 8.51(2 \mathrm{H}, \mathrm{d}, J=7.7 \mathrm{~Hz}, 2$ '$\left.\mathrm{H}, 6^{\prime}-\mathrm{H}\right), 8.18(1 \mathrm{H}, \mathrm{d}, J=8.51 \mathrm{~Hz}, 8-\mathrm{H}), 7.70-7.77(2 \mathrm{H}, \mathrm{m}, 5-\mathrm{H}, 7-\mathrm{H}), 7.58(2 \mathrm{H}, \mathrm{t}, J=7.6 \mathrm{~Hz}$, 5"-H, 3"'-H), 7.32-7.40 (3H, m, 6-H, 2H arom), 7.28 (1H, t, J = 7.4 Hz, 4'-H), 7.12 (2H, d, J = 8.5 $\left.\mathrm{Hz}, \mathrm{H}_{\text {arom }}\right), 3.95$ (3H, s, OMe), 2.20 (3H, s, 3-Me).

3,6-Dimethyl-4-(4'-methoxyphenyl)-1-phenyl-1H-pyrazolo[3,4-b]quinoline (8c). Yellow 
crystals, 32\%, m..p. $188-190{ }^{\circ} \mathrm{C}$ (Lit. $\left.{ }^{5} 188-9{ }^{\circ} \mathrm{C}\right) .{ }^{1} \mathrm{H}$ NMR $\left(300 \mathrm{MHz}, \mathrm{CDCl}_{3}\right): \delta 8.51(2 \mathrm{H}, \mathrm{d}, J=$ $7.7 \mathrm{~Hz}, 2^{\prime}-\mathrm{H}, 6$ '-H), 8.08 (1H, d, J = 8.8 Hz, 8-H), 7.54-7.56 (3H, m, 7-H, 2H arom), 7.53 (1H, s, 5-H), $7.36\left(2 \mathrm{H}, \mathrm{d}, J=8.5 \mathrm{~Hz}, \mathrm{H}_{\text {arom }}\right), 7.26\left(1 \mathrm{H}, \mathrm{t}, J=7.4 \mathrm{~Hz}, \mathrm{H}_{\text {arom }}\right), 7.11(2 \mathrm{H}, \mathrm{d}, J=8.5 \mathrm{~Hz}$, $\left.\mathrm{H}_{\text {arom }}\right), 3.95$ (3H, s, OMe), 2.43 (3H, s, 6-Me), 2.16 (3H, s, 3-Me).

6-tert-Butyl-4-(4'-methoxyphenyl)-3-methyl-1-phenyl-1H-pyrazolo[3,4-b]quinoline (8d). Light yellow needles, $30.5 \%$ m.p. $210-1{ }^{\circ} \mathrm{C}$ (Lit. $\left.{ }^{5} 210-1{ }^{\circ} \mathrm{C}\right) .{ }^{1} \mathrm{H}$ NMR $\left(300 \mathrm{MHz}, \mathrm{CDCl}_{3}\right): \delta 8.50$ $\left(2 \mathrm{H}, \mathrm{d}, J=7.7 \mathrm{~Hz}, 2^{\prime}-\mathrm{H}, 6\right.$ ' $\left.-\mathrm{H}\right), 8.12(1 \mathrm{H}, \mathrm{d}, J=9.0 \mathrm{~Hz}, 8-\mathrm{H}), 7.85$ (1H, dd, $J=9.0,1.9 \mathrm{~Hz}, 7-$ $\mathrm{H}), 7.67(1 \mathrm{H}, \mathrm{d}, J=1.9 \mathrm{~Hz}, 5-\mathrm{H}), 7.58\left(2 \mathrm{H}, \mathrm{t}, J=7.5 \mathrm{~Hz}, \mathrm{H}_{\text {arom }}\right), 7.39(2 \mathrm{H}, \mathrm{d}, J=8.51 \mathrm{~Hz}$, $\left.\mathrm{H}_{\text {arom }}\right), 7.26\left(1 \mathrm{H}, \mathrm{t}, J=7.4 \mathrm{~Hz}, \mathrm{H}_{\text {arom }}\right), 7.12\left(2 \mathrm{H}, \mathrm{d}, J=8.5 \mathrm{~Hz}, \mathrm{H}_{\text {arom }}\right), 3.96(3 \mathrm{H}, \mathrm{s}, \mathrm{OMe}), 2.19$ (3H, s, 3-Me), $1.32(9 \mathrm{H}, \mathrm{s}, 9 \mathrm{H}$, tert-Bu).

6-Fluoro-4-(4'-methoxyphenyl)-3-methyl-1-phenyl-1H-pyrazolo [3,4-b]quinoline

(8e). Yellow crystals, 30\%, m.p. $185-6{ }^{\circ} \mathrm{C}$ (Lit. $\left.{ }^{5} 184-6{ }^{\circ} \mathrm{C}\right) .{ }^{1} \mathrm{H}$ NMR $\left(300 \mathrm{MHz}, \mathrm{CDCl}_{3}\right): \delta 8.47(2 \mathrm{H}$, d, $\left.J=7.4 \mathrm{~Hz}, 2^{\prime}-\mathrm{H}, 6^{\prime}-\mathrm{H}\right), 8.15(1 \mathrm{H}, \mathrm{dd}, J \mathrm{HH}=9.3 \mathrm{~Hz}, J \mathrm{HF}=5.5 \mathrm{~Hz}, 8-\mathrm{H}), 7.46-7.60$ (3H, m, 5H, 3"-H, 5"-H), 7.30-7.40 (3H, m, 7-H, 3"-H, 5"-H), 7.27 (1H, t, J = 7.4 Hz, 4'-H), 7.10 (2H, d, $J=8.5 \mathrm{~Hz}, 2$ "-H, 6"-H), 3.94 (3H, s, OMe), 2.17 (3H, s, 3-Me).

6-Chloro-4-(4'-methoxyphenyl)-3-methyl-1-phenyl-1H-pyrazolo[3,4-b]quinoline

(8f). Yellow crystals, 29\%, m.p. $204-6{ }^{\circ} \mathrm{C}$ (Lit. $\left.{ }^{5} 204-5{ }^{\circ} \mathrm{C}\right) .{ }^{1} \mathrm{H} \mathrm{NMR}\left(300 \mathrm{MHz}, \mathrm{CDCl}_{3}\right): \delta 8.45(2 \mathrm{H}$, d, $\left.J=7.4 \mathrm{~Hz}, 2^{\prime}-\mathrm{H}, 6^{\prime}-\mathrm{H}\right), 8.09$ (1H, d, $\left.J=9.1 \mathrm{~Hz}, 8-\mathrm{H}\right), 7.70(1 \mathrm{H}, \mathrm{d}, J=2.2 \mathrm{~Hz}, 5-\mathrm{H}), 7.64(1 \mathrm{H}$, $\mathrm{dd}, J=9.1,2.4 \mathrm{~Hz}, 7-\mathrm{H}), 7.55\left(2 \mathrm{H}, \mathrm{t}, J=7.4 \mathrm{~Hz}, \mathrm{H}_{\text {arom }}\right), 7.35\left(2 \mathrm{H}, \mathrm{d}, J=8.8 \mathrm{~Hz}, \mathrm{H}_{\text {arom }}\right), 7.27$ $\left(1 \mathrm{H}, \mathrm{t}, J=7.4 \mathrm{~Hz}, \mathrm{H}_{\text {arom }}\right), 7.11\left(2 \mathrm{H}, \mathrm{d}, J=8.8 \mathrm{~Hz}, \mathrm{H}_{\text {arom }}\right), 3.96(3 \mathrm{H}, \mathrm{s}, \mathrm{OMe}), 2.17$ (3H, s, 3-Me).

6-Bromo-4-(4'-methoxyphenyl)-3-methyl-1-phenyl-1H-pyrazolo $[3,4-b] q u i n o l i n e$

(8g). Yellow crystals, 30\%, m.p. $182-3{ }^{\circ} \mathrm{C}$ (Lit. $\left.{ }^{5} 182-3{ }^{\circ} \mathrm{C}\right) .{ }^{1} \mathrm{H} \mathrm{NMR}\left(300 \mathrm{MHz}, \mathrm{CDCl}_{3}\right): \delta 8.46(2 \mathrm{H}$, $\left.\mathrm{d}, J=7.7 \mathrm{~Hz}, \mathrm{H}_{\text {arom }}\right), 7.96(1 \mathrm{H}, \mathrm{d}, J=9.1,2.2 \mathrm{~Hz}, 8-\mathrm{H}), 7.83(1 \mathrm{H}, \mathrm{d}, J=2.2 \mathrm{~Hz}, 5-\mathrm{H}), 7.7(1 \mathrm{H}$, $\mathrm{dd}, J=9.1 \mathrm{~Hz}, 7-\mathrm{H}), 7.53\left(2 \mathrm{H}, \mathrm{t}, J=7.5 \mathrm{~Hz}, \mathrm{H}_{\text {arom }}\right), 7.33\left(2 \mathrm{H}, \mathrm{d}, J=6.6 \mathrm{~Hz}, \mathrm{H}_{\text {arom }}\right), 7.26(1 \mathrm{H}, \mathrm{t}, J$ $\left.=7.4 \mathrm{~Hz}, \mathrm{H}_{\text {arom }}\right), 7.09\left(2 \mathrm{H}, \mathrm{d}, J=6.6 \mathrm{~Hz}, \mathrm{H}_{\text {arom }}\right), 3.94(3 \mathrm{H}, \mathrm{s}, \mathrm{OMe}), 2.12(3 \mathrm{H}, \mathrm{s}, 3-\mathrm{Me})$.

4-(4'-Methoxybenzal)-3-methyl-1-phenylpyrazolin-5-one (9). Colourless crystals, 31-32\%, m.p. 166-8 ${ }^{\circ} \mathrm{C}$. Anal. calc. for $\mathrm{C}_{18} \mathrm{H}_{18} \mathrm{~N}_{2} \mathrm{O}_{2}$ : C $73.45 \mathrm{H} 6.16 \mathrm{~N} 9.52$; found $\mathrm{C} 73.30 \mathrm{H} 6.06 \mathrm{~N}$ 9.41 .

Pyrazolo[3,4-b]quinolines from the reaction of p-substituted anilines with 4-(4'methylphenyl)-pyrazolin-5-ones

4-(4'-Methylphenyl)-3-methyl-1-phenyl-1H-pyrazolo[3,4-b]quinoline (8h). Yellow crystals, $32 \%$, m.p. $206-7{ }^{\circ} \mathrm{C}, \lambda_{\text {em }}($ cyclohexane $)=437 \mathrm{~nm} .{ }^{1} \mathrm{H}$ NMR $\left(300 \mathrm{MHz}, \mathrm{CDCl}_{3}\right): \delta 8.51(2 \mathrm{H}, \mathrm{d}, J$ $\left.=8.7 \mathrm{~Hz}, 2^{\prime}-\mathrm{H}, 6^{\prime}-\mathrm{H}\right), 8.21(1 \mathrm{H}, \mathrm{d}, J=9.05 \mathrm{~Hz}, 8-\mathrm{H}), 7.75(1 \mathrm{H}, \mathrm{d}, J=8.7 \mathrm{~Hz}, 5-\mathrm{H}), 7.74(1 \mathrm{H}, \mathrm{t}, J$ $=8.65 \mathrm{~Hz}, 6-\mathrm{H}), 7.56(2 \mathrm{H}, \mathrm{t}, J=8.0 \mathrm{~Hz}, 3$ '-H, 5'-H), 7.32-7.42 (1H, m, 5-H), $7.4(2 \mathrm{H}, \mathrm{d}, J=8.0$ Hz, 3"-H, 5"-H), 7.34 (2H, d, J=8.0 Hz, 2"-H, 6"-H), 7.28 (1H, t, J= 7.4 Hz, 4'-H), 2.52 (3H, s,

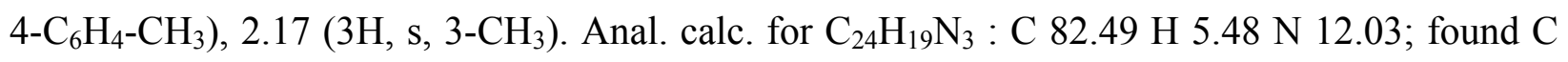
$82.38 \mathrm{H} 5.26 \mathrm{~N} 12.17$.

3,6-Dimethyl-4-(4'-methylphenyl)-1-phenyl-1H-pyrazolo[3,4-b]quinoline $\quad$ (8i). Yellow crystals, $31 \%$, m.p. $197-8{ }^{\circ} \mathrm{C}$. $\lambda_{\text {em }}$ (cyclohexane) $=438.5 \mathrm{~nm} .{ }^{1} \mathrm{H}$ NMR $\left(300 \mathrm{MHz}, \mathrm{CDCl}_{3}\right): \delta$ 
$8.50\left(2 \mathrm{H}, \mathrm{d}, J=8,7 \mathrm{~Hz}, 2^{\prime}-\mathrm{H}, 6^{\prime}-\mathrm{H}\right), 8.11(1 \mathrm{H}, \mathrm{d}, J=8.7 \mathrm{~Hz}, 8-\mathrm{H}), 7.57(1 \mathrm{H}, \mathrm{d}, J=8.76 \mathrm{~Hz}, 7-$ H), 7.55 (2H, t, $J=8.0 \mathrm{~Hz}, 3$ '-H, 5'-H), 7.47 (1H, s, 5-H), 7.40 (2H, d, J=8.23 Hz, 3'-H, 5'H), 7.33(2H, d, $J=8.23 \mathrm{~Hz}, 2$ "'-H, 6"-H), 7.27 (1H, t, $J=7.4 \mathrm{~Hz}, 4$ '-H), 2.53 (3H, s, 4-C $\mathrm{H}_{4}-$ $\left.\mathrm{CH}_{3}\right), 2.43\left(3 \mathrm{H}, \mathrm{s}, 6-\mathrm{CH}_{3}\right), 2.14\left(3 \mathrm{H}, \mathrm{s}, 3-\mathrm{CH}_{3}\right)$. Anal. calc. for $\mathrm{C}_{25} \mathrm{H}_{21} \mathrm{~N}_{3}: \mathrm{C} 82.62 \mathrm{H} 5.82 \mathrm{~N}$ 11.56; found $\mathrm{C} 82.51 \mathrm{H} 5.67 \mathrm{~N} 11.32$.

6-Ethyl-3-methyl-4-(4'-methylphenyl)-1-phenyl-1H-pyrazolo[3,4-b]quinoline (8j). Yellow crystals, 30\%, m.p. $158-9{ }^{\circ} \mathrm{C}$. $\lambda_{\text {em }}$ (cyclohexane) $=437 \mathrm{~nm} .{ }^{1} \mathrm{H}$ NMR $\left(300 \mathrm{MHz}, \mathrm{CDCl}_{3}\right): \delta 8.53$ $\left(2 \mathrm{H}, \mathrm{d}, J=8.7 \mathrm{~Hz}, 2^{\prime}-\mathrm{H}, 6{ }^{\prime}-\mathrm{H}\right), 8.13(1 \mathrm{H}, \mathrm{d}, J=8.8 \mathrm{~Hz}, 8-\mathrm{H}), 7.62$ (1H, d, J=8.8 Hz, 7-H), 7.55 (2H, t, $J=8 \mathrm{~Hz}, 3$ '-H, 5'-H), 7.49 (1H, s, J=2 Hz, 5-H), 7.40 (2H, d, J = $7.8 \mathrm{~Hz}, 3$ '-H, 5'H), 7.34 (2H, d, J=7.8 Hz, 2"'-H, 6"-H), 7.27 (1H, t, J=7.4 Hz, 4'-H), 2.74 (2H, q, J = $7.5 \mathrm{~Hz}$, 6- $\left.\mathrm{CH}_{2} \mathrm{CH}_{3}\right), 2.54\left(3 \mathrm{H}, \mathrm{s}, 4-\mathrm{C}_{6} \mathrm{H}_{4}-\mathrm{CH}_{3}\right), 2.16\left(3 \mathrm{H}, \mathrm{s}, 3-\mathrm{CH}_{3}\right), 1.25(3 \mathrm{H}, \mathrm{t}, J=7.5 \mathrm{~Hz}, 6-$ $\mathrm{CH}_{2} \mathrm{CH}_{3}$ ). Anal. calc. for $\mathrm{C}_{26} \mathrm{H}_{23} \mathrm{~N}_{3}$ : C $82.736 .14 \mathrm{~N} 11.13$; found $\mathrm{C} 82.52 \mathrm{H} 6.21 \mathrm{~N} 11.31$.

6-n-Butyl-3-methyl-4-(4'-methylphenyl)-1-phenyl-1H-pyrazolo[3,4-b]quinoline (8k). Yellow needles, 30\%, m.p. $246-7{ }^{\circ} \mathrm{C} . \lambda_{\text {em }}$ (cyclohexane) $=437 \mathrm{~nm} .{ }^{1} \mathrm{H}$ NMR $\left(300 \mathrm{MHz}, \mathrm{CDCl}_{3}\right): \delta 8.49$ $\left(2 \mathrm{H}, \mathrm{d}, J=8.7 \mathrm{~Hz}, 2^{\prime}-\mathrm{H}, 6^{\prime}-\mathrm{H}\right), 8.13(1 \mathrm{H}, \mathrm{d}, J=8.8 \mathrm{~Hz}, 8-\mathrm{H}), 7.61(1 \mathrm{H}, \mathrm{d}, J=8.8 \mathrm{~Hz}, J '=1.9 \mathrm{~Hz}$, 7-H), $7.55\left(2 \mathrm{H}, \mathrm{t}, J=8.0 \mathrm{~Hz}, 3^{\prime}-\mathrm{H}, 5^{\prime}-\mathrm{H}\right), 7.47(1 \mathrm{H}, \mathrm{s}, J=1.9 \mathrm{~Hz}, 7.40,5-\mathrm{H}), 7.40$ (2H, d, $J=$ $8.2 \mathrm{~Hz}, 3$ 3"-H, 5"-H), 7.33 (2H, d, J=8.2 Hz, 2"-H, 6"-H), 7.25 (1H, t, J = 7.4 Hz, 4'-H), 2.68 $\left(2 \mathrm{H}, \mathrm{t}, 6-\mathrm{CH}_{2} \mathrm{CH}_{2} \mathrm{CH}_{2} \mathrm{CH}_{3}\right), 2.54\left(3 \mathrm{H}, \mathrm{s}, 4-\mathrm{C}_{6} \mathrm{H}_{4}-\mathrm{CH}_{3}\right), 2.14\left(3 \mathrm{H}, \mathrm{s}, 3-\mathrm{CH}_{3}\right), 1.60$ (2H, pent, 6$\mathrm{CH}_{2} \mathrm{CH}_{2} \mathrm{CH}_{2} \mathrm{CH}_{3}$ ), 1.34 (2H, sext, 6- $\left.\mathrm{CH}_{2} \mathrm{CH}_{2} \mathrm{CH}_{2} \mathrm{CH}_{3}\right), 0.90\left(3 \mathrm{H}, \mathrm{t}, 3 \mathrm{H}, 6-\mathrm{CH}_{2} \mathrm{CH}_{2} \mathrm{CH}_{2} \mathrm{CH}_{3}\right.$ ).

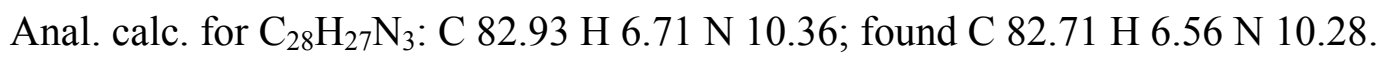

6-Fluoro-3-methyl-4-(4'-methylphenyl)-1-phenyl-1H-pyrazolo[3,4-b]quinoline (8I). Light yellow crystals, 30\%, m.p. $185-7{ }^{\circ} \mathrm{C}$. $\lambda_{\text {em }}$ (cyclohexane) $=444.5 \mathrm{~nm} .{ }^{1} \mathrm{H}$ NMR $(300 \mathrm{MHz}$, $\left.\mathrm{CDCl}_{3}\right): \delta 8.47\left(2 \mathrm{H}, \mathrm{d}, \mathrm{J}=8.7 \mathrm{~Hz}, 2^{\prime}-\mathrm{H}, 6^{\prime}-\mathrm{H}\right), 8.18\left(1 \mathrm{H}, \mathrm{dd}, \mathrm{J}_{\mathrm{HH}}=9.4, \mathrm{~J}_{\mathrm{HF}}=5.4 \mathrm{~Hz}, 8-\mathrm{H}\right), 7.24-7.60$ $\left(9 \mathrm{H}, \mathrm{m}, \mathrm{H}_{\text {arom }}\right), 2.52\left(3 \mathrm{H}, \mathrm{s}, 4-\mathrm{C}_{6} \mathrm{H}_{4}-\mathrm{CH}_{3}\right), 2.16\left(3 \mathrm{H}, \mathrm{s}, 3-\mathrm{CH}_{3}\right)$. Anal. calc. for $\mathrm{C}_{24} \mathrm{H}_{18} \mathrm{FN}_{3}$ : C 78.46 H 4.94 N 11.44; found C 78.31 H 4.76 N 11.30.

6-Chloro-3-methyl-4-(4'-methylphenyl)-1-phenyl-1H-pyrazolo[3,4-b]quinoline (8m). Yellow needles, 30\%, m.p. $201-3^{\circ} \mathrm{C} . \lambda_{\mathrm{em}}$ (cyclohexane) $=446 \mathrm{~nm} .{ }^{1} \mathrm{H}$ NMR $\left(300 \mathrm{MHz}, \mathrm{CDCl}_{3}\right): \delta 8.46$ $\left(2 \mathrm{H}, \mathrm{d}, J=8.7 \mathrm{~Hz}, 2^{\prime}-\mathrm{H}, 6^{\prime}-\mathrm{H}\right), 8.12(1 \mathrm{H}, \mathrm{d}, J=9.0 \mathrm{~Hz}, 8-\mathrm{H}), 7.68(1 \mathrm{H}, \mathrm{s}, J$ ' $=2.3 \mathrm{~Hz}, 5-\mathrm{H})$, $7.64\left(1 \mathrm{H}, \mathrm{d}, J=9.0, J^{\prime}=2.3 \mathrm{~Hz}, 7-\mathrm{H}\right), 7.55\left(2 \mathrm{H}, \mathrm{t}, J=8.0 \mathrm{~Hz}, 3^{\prime}-\mathrm{H}, 5\right.$ ' $\left.-\mathrm{H}\right), 7.40$ (2H, d, $J=8.1$ $\mathrm{Hz}, 3$ 3“-H, 5“-H), $7.31\left(1 \mathrm{H}, \mathrm{d}, J=8.1 \mathrm{~Hz}, 2 “-\mathrm{H}, 66^{\prime \prime}-\mathrm{H}\right), 7.28(1 \mathrm{H}, \mathrm{t}, J=7.4 \mathrm{~Hz}, 4$ '-H), 2.53 (s, 3H, 4- $\left.\mathrm{C}_{6} \mathrm{H}_{4}-\mathrm{CH}_{3}\right), 2.14\left(\mathrm{~s}, 3 \mathrm{H}, 3-\mathrm{CH}_{3}\right)$. Anal. calc. for $\mathrm{C}_{24} \mathrm{H}_{18} \mathrm{ClN}_{3}$ : C $75.09 \mathrm{H} 4.73 \mathrm{~N} \mathrm{10.95}$; found C $75.24 \mathrm{H} 4.86 \mathrm{~N} 10.87$.

6-Bromo-3-methyl-4-(4'-methylphenyl)-1-phenyl-1H-pyrazolo[3,4-b]quinoline (8n). Yellow crystals, $32 \%$, m.p. $205-6{ }^{\circ} \mathrm{C}$. $\lambda_{\text {em }}$ (cyclohexane) $=447 \mathrm{~nm} .{ }^{1} \mathrm{H}$ NMR $\left(300 \mathrm{MHz}, \mathrm{CDCl}_{3}\right): \delta 8.46$ $\left(2 \mathrm{H}, \mathrm{d}, J=8.7 \mathrm{~Hz}, 2^{\prime}-\mathrm{H}, 6^{\prime}-\mathrm{H}\right), 8.04(1 \mathrm{H}, \mathrm{d}, J=9.1 \mathrm{~Hz}, 8-\mathrm{H}), 7.86\left(1 \mathrm{H}, \mathrm{s}, J^{\prime}=2.0 \mathrm{~Hz}, 5-\mathrm{H}\right)$, $7.75\left(1 \mathrm{H}, \mathrm{d}, J=9.1, J^{\prime}=2.0 \mathrm{~Hz}, 7-\mathrm{H}\right), 7.55\left(2 \mathrm{H}, \mathrm{t}, J=8.0 \mathrm{~Hz}, 3^{\prime}-\mathrm{H}, 5^{\prime}-\mathrm{H}\right), 7.40$ (2H, d, $J=8.1$ $\mathrm{Hz}, 3$ 3“-H, 5“-H), $7.31(2 \mathrm{H}, \mathrm{d}, J=8.1 \mathrm{~Hz}, 2$ '“-H, 6“- $\mathrm{H}), 7.28(1 \mathrm{H}, \mathrm{t}, J=7.4 \mathrm{~Hz}, 4$ ' $-\mathrm{H}), 2.53$ (3H, s, 4- $\left.\mathrm{C}_{6} \mathrm{H}_{4}-\mathrm{CH}_{3}\right), 2.13\left(3 \mathrm{H}, \mathrm{s}, 3-\mathrm{CH}_{3}\right)$. Anal. calc. for $\mathrm{C}_{24} \mathrm{H}_{18} \mathrm{BrN}_{3}: \mathrm{C} 67.30 \mathrm{H} 4.24 \mathrm{~N} 9.81$; found C $67.05 \mathrm{H} 4.34 \mathrm{~N} 9.76$.

6-Methoxy-3-methyl-4-(4'-methylphenyl)-1-phenyl-1H-pyrazolo[3,4-b]quinoline 
(8o). Yellow crystals, 30\%, m.p. $231-2{ }^{\circ} \mathrm{C} . \lambda_{\mathrm{em}}$ (cyclohexane) $=448.5 \mathrm{~nm} .{ }^{1} \mathrm{H}$ NMR $(300 \mathrm{MHz}$, $\left.\mathrm{CDCl}_{3}\right): \delta 8.49\left(2 \mathrm{H}, \mathrm{d}, J=8.7 \mathrm{~Hz}, 2^{\prime}-\mathrm{H}, 6^{\prime}-\mathrm{H}\right), 8.11(1 \mathrm{H}, \mathrm{d}, J=9.2 \mathrm{~Hz}, 8-\mathrm{H}), 7.54(2 \mathrm{H}, \mathrm{t}, J=$ $7.97 \mathrm{~Hz}, 3$ '-H, 5'-H), 7.43 (1H, d, J=9.2 Hz, J' = 2.8 Hz, 7-H), 7.36-7.37 (4H, 2“-H, 3“--H, 5“$\left.\mathrm{H}, 66^{\prime \prime}-\mathrm{H}\right), 7.26\left(1 \mathrm{H}, \mathrm{t}, J=7.4 \mathrm{~Hz}, 4\right.$ '-H), $6.94(1 \mathrm{H}, \mathrm{s}, J$ ' $=2.8 \mathrm{~Hz}, 5-\mathrm{H}), 3.73\left(3 \mathrm{H}, \mathrm{s}, 6-\mathrm{OCH}_{3}\right)$, $2.52\left(3 \mathrm{H}, \mathrm{s}, 4-\mathrm{C}_{6} \mathrm{H}_{4}-\mathrm{CH}_{3}\right), 2.14\left(3 \mathrm{H}, \mathrm{s}, 3-\mathrm{CH}_{3}\right)$. Anal. calc. for $\mathrm{C}_{25} \mathrm{H}_{21} \mathrm{~N}_{3} \mathrm{O}: \mathrm{C} 79.13 \mathrm{H} 5.58 \mathrm{~N}$ 11.07; found C 79.01 H 5.51 N 10.87.

3-Methyl-4-(4'-methylphenyl)-6-phenoxy-1-phenyl-1H-pyrazolo[3,4-b]quinoline (8p). Yellow crystals, $30 \%$, m.p. $158-9{ }^{\circ} \mathrm{C}$. $\lambda_{\text {em }}$ (cyclohexane) $=446.5 \mathrm{~nm} .{ }^{1} \mathrm{H} \mathrm{NMR}(300 \mathrm{MHz}$, $\left.\mathrm{CDCl}_{3}\right): \delta 8.50\left(2 \mathrm{H}, \mathrm{d}, J=8.0 \mathrm{~Hz}, 2^{\prime}-\mathrm{H}, 6^{\prime}-\mathrm{H}\right), 8.20(1 \mathrm{H}, \mathrm{d}, J=9.1 \mathrm{~Hz}, 8-\mathrm{H}), 7.46-7.61(3 \mathrm{H}, \mathrm{m}$, $\left.\mathrm{H}_{\text {arom }}\right), 7.24-7.38\left(8 \mathrm{H}, \mathrm{m}, \mathrm{H}_{\text {arom }}\right), 7.07(1 \mathrm{H}, \mathrm{t}, J=7.3 \mathrm{~Hz}, 4$ '"'-H in 6-OPh), $6.97(2 \mathrm{H}, \mathrm{d}, J=8.5$ $\mathrm{Hz}, \mathrm{H}_{\text {arom }}$ ), 6.97 (2H, d, 2"' -H and 6"'-H in 6-OPh), 2.48 (3H, s, 4-C $\left.6 \mathrm{H}_{4}-\mathrm{CH} 3\right), 2.17$ (3H, s, 3$\mathrm{CH}_{3}$ ). Anal. calc. for $\mathrm{C}_{30} \mathrm{H}_{23} \mathrm{~N}_{3} \mathrm{O}: \mathrm{C} 81.61 \mathrm{H} 5.25 \mathrm{~N}$ 9.52; found $\mathrm{C} 81.52 \mathrm{H} 5.23 \mathrm{~N} 9.43$.

\section{Acknowledgements}

In the period of 1969/1970 the corresponding author has received from Dr. R.A. Abramovitch one-year postdoctoral fellowship. Our boss - postdoc relations turned soon into friendship and extended cooperation. With this paper we are paying our tribute to Dr. Abramovitch. We should like to show that the seed he put into scientific soil during his volcanic activity yielded a crop also in Poland. Thanks a lot! Financial support from the Polish Research Council (Grant No KBN 8T11B 075 18) is gratefully acknowledged.

\section{References}

1. Michaelis, A. Liebigs Ann. 1911, 385, 24.

2. Danel, A.; Chaczatrian, K.; Chaczatrian, G.; Tomasik, P. ARKIVOC, 2000, 1, 51.

3. Tomasik, P.; Tomasik, D.; Abramovitch, R. A. J. Heterocycl. Chem. 1983, 20, 1539.

4. Danel, A. Ph.D. Thesis; Jagiellonian University; Cracow 1996.

5. Tao, Y. T.; Balasubramaniam, E.; Danel, A.; Wisła, A.; Tomasik, P. J. Mater. Chem. 2001, 11,768 .

6. Mahmoud, M. R.; El-Kashef, H. S.; Abd El-Hamide, R. Spectrochim. Acta 1981, 37A, 519.

7. Jaffe, H. H.; Orchin, M. Theory and Applications of Ultraviolet and Visible Spectroscopy Wiley: New York, 1964; Ch. 8. 